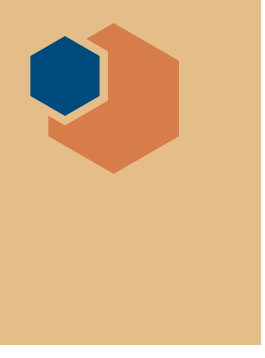

\section{Mandates for public access to publications and data on the horizon for US researchers}

www.ostp.gov

$\mathbf{U}$ S researchers will soon face requirements to publicly share both the peer-reviewed publications and the digital data that result from their federally funded work. In mid-February, the White House Office of Science and Technology Policy (OSTP) issued a memorandum that gave federal agencies with over $\$ 100$ million in annual research and development expenditures a six-month time frame for developing plans to increase public access to federally funded research results. The memo argues that these public access policies will accelerate scientific breakthroughs and enhance economic growth and job creation.

The US government has been developing its public access objectives for several years, gathering input from the National Science and Technology Council and public consultation. The OSTP memo follows from a requirement in the America COMPETES Reauthorization Act of 2010 (P.L. 111-358) for policies to improve the quality, access, dissemination, and long-term stewardship of federally supported research results. The United States is not the only country making the move to public access. The United Kingdom implemented new open access $(\mathrm{OA})$ policies for peer-reviewed publications on April 1, 2013, and the European Commission has plans to expand its $\mathrm{OA}$ requirements as well.

For publications, the memo requires agencies to "ensure that the public can read, download, and analyze in digital form final peer-reviewed manuscripts or final published documents." It recommends a 12-month post-publication embargo period before research papers must be made publicly available, but allows agencies to tailor the time frame to accommodate the needs of different disciplines. The National Institutes of Health already mandates that publications resulting from its funding be deposited in the PubMed Central database within 12 months of publication, and some journals, such as Science, make all of their articles freely available after 12 months.

Most journals already offer at least the option for OA publication, either under the Green or Gold OA model. The Green OA model allows authors to deposit articles in non-commercial repositories like arXiv.org, or to post an article to their own website, while the Gold OA model allows free access directly from the publisher's website, with publication costs covered by the author through grant funds or means other than traditional subscriptions.

However, several publishers that depend heavily on subscription revenue to cover their expenses are concerned that a 12-month embargo would jeopardize their financial sustainability. The Institute of Electrical and Electronics Engineers, for example, has noted that $85 \%$ of articles retrieved from its digital library are older than 12 months. To remain sustainable, stakeholders advocate that agency procedures accommodate the economic implications of various public access models.

The memo leaves open the question of how public access will be achieved, requiring only that agency procedures "facilitate easy public search, analysis of, and access to" the publications. If a repository approach is chosen, the management of this infrastructure could be centralized within the federal government or distributed in separate repositories managed by institutions, publishers, professional societies, or other third parties. Ideal functionalities of a repository include capabilities for bulk downloading, text mining, and computational analysis, as well as interoperability with other repositories and long-term stewardship and preservation of the work.

The OSTP memo requires that data sets used to support scholarly publications "should be stored and publicly accessible to search, retrieve, and analyze," so long as they are non-confidential and non-proprietary. The memo does not specify the time period before data must be made publicly available, but stakeholder recommendations have ranged from immediately after the data have been gathered, to after an embargoed publication period, to upon completion of the relevant supporting grant.

Currently, data access and analysis are hampered by inconsistencies

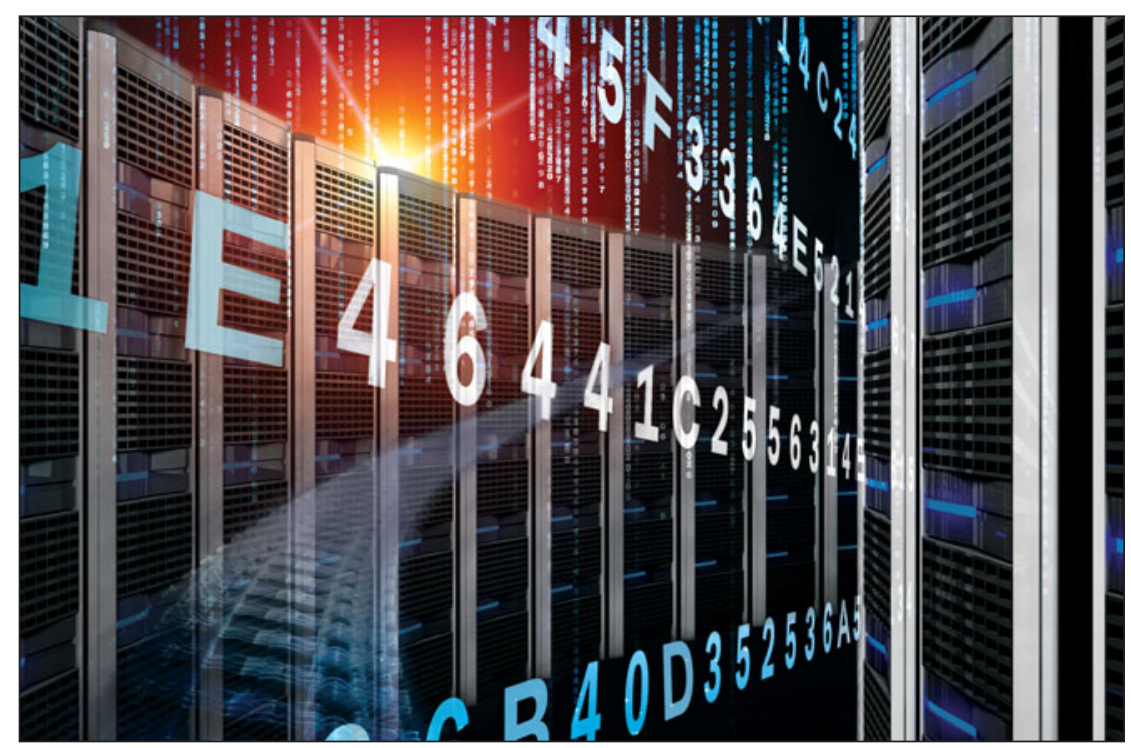


in formatting, nomenclature, metadata standards, and approaches for storage. Implementation of any plan will need to address these issues. As with publications, an infrastructure for housing data contributions has not been determined and may require considerable development to include full functionality, such as search functions, mining, and interoperability with other repositories. Additionally, stakeholders recommend a standardized data attribution system along the lines of the Digital Object Identifier System, ideally linked to the relevant publication.

A significant and as-yet unanswered question is how these policies will be funded. The OSTP memo recommends leveraging existing archives and fostering public-private partnerships to maximize efficiencies. It also calls for agencies to identify resources within their existing budgets to implement the plans and ensure compliance with their policies. However, it seems likely that full implementation and compliance will require more resources than are available in existing budgets.

The cost of making data publicly accessible is unknown. The OSTP memo requires that agencies allow the inclusion of appropriate costs for data management and access in grant proposals, but in the case of terabyte-scale data sets common among some computational scientists, this could consume a significant portion of a grant budget. The short-term costs associated with databases include storage and distribution bandwidth, but agency plans should also provide contingencies for long-term costs such as preservation and potential migration. Some aspects of the infrastructure and expenses should be more manageable in the near term, as the memo stipulates that these public access requirements will only apply to manuscripts submitted for publication and data generated after the effective date of any implemented agency policy.

As the August 22, 2013 deadline for agencies to deliver their draft plans to OSTP fast approaches, the policies are currently being discussed in many forums, including National Research Council public comment sessions which took place in May. While many details associated with carrying out the OSTP memo's objectives are in development, one thing seems certain - proper implementation of increased public access to publications and data will require significant resources in a time of constrained budgets.

Ashley A. White
Long-term partnership to help UK compete in global

nuclear industry www.gov.uk

A Nuclear Industrial Strategy, developed by the UK government and industry, has been published this year to enable the United Kingdom to seize the opportunities for economic growth in the nuclear industry. It covers the whole of the nuclear market — new build, waste management and decommissioning, fuel cycle services, operations, and maintenance.

The strategy is being overseen by a Nuclear Industry Council, co-chaired by ministers and industry. It includes a wide range of commitments, including:

- $£ 15$ million for a new world-class National Nuclear Users Facility for universities and companies carrying out research into nuclear technology. The facility will have centers at the National Nuclear Laboratory at Sellafield, the Culham Centre for Fusion Energy in Oxfordshire, and the University of Manchester's Dalton Cumbrian Facility.

- 36 nuclear research and development $(R \& D)$ projects have won $£ 18$ million worth of support from a Technology Strategy Board competition, which will leverage in a further
$£ 13$ million of private sector investment. This includes a consortium led by OC Robotics in Bristol who have received almost $£ 6$ million to develop their LaserSnake technology - a robot-controlled laser cutting tool that can be used as part of nuclear decommissioning projects.

- $£ 12.5$ million to join the Jules Horowitz Test Reactor Program, which is being constructed in France. The reactor will provide the United Kingdom with a radiation testing facility to develop future advance nuclear fuels.

- The government spent $£ 66$ million in 2011 on nuclear R\&D and will keep under review its level of future expenditure. It is keen to explore opportunities to back future reactor designs, including the feasibility of launching a Small Modular Reactor R\&D program to ensure that the United Kingdom is a key partner of any new reactor design for the global market.

The strategy is based on the recommendations of the Ad-Hoc Nuclear
Research and Development Advisory Board. The Advisory Board was established to oversee work on an industrial vision statement, an $R \& D$ roadmap, a review of the $R \& D$ landscape, and a long-term nuclear energy strategy.

The Board's recommendations recognize that nuclear power will continue to play an important role in the Nuclear Industrial Strategy to 2050 and beyond, and that a wide range of technologies may be required to meet the challenges of an expanded demand for nuclear power.

Sir John Beddington, the government's Chief Scientific Advisor, said, "I am convinced that nuclear power will play a pivotal role in the UK's energy future. The requirement for nuclear power may exceed current plans for new build, perhaps substantially. It's therefore crucial that we keep a wide range of technological options open so that we are able to meet this potential demand in a safe and sustainable manner."

Long-term strategies are being developed by the UK government in partnership with industry in key sectors including oil and gas, education, and the information economy. 